\title{
Enhanced the Performance of Fluorescence Gas Sensor of Porphyrin Dye By Using $\mathrm{TiO}_{2}$ Nanoparticles
}

\author{
N. H. Yusoff ${ }^{a}$, M. M. Salleh ${ }^{b}$ and M. Yahaya ${ }^{c}$ \\ Institute of Microengineering and Nanoelectronics \\ Universiti Kebangsaan Malaysia, 43600 Bangi, Selangor, Malaysia \\ anhudayusoff@yahoo.com, bmms@ukm.my, cmyahya@ukm.my
}

Keywords: porphyrin dye, $\mathrm{TiO}_{2}$ nanoparticles coated with dye, fluorescence gas sensor.

\begin{abstract}
Fluorescence gas sensor was developed to identify the presence of volatile organic compounds by using porphyrin dye thin film. The porphyrin dye used was iron (III) mesotetraphenylporphine chloride. The porphyrin thin film was deposited on quartz substrate using selfassembly through dip coating technique. The sensing properties of the thin films toward volatile organic compounds; ethanol, acetone and 2-propanol were studied using luminescence spectrometer. In presence of air and volatile organic compounds, thin films produced different emission spectra and ease for chemical identification process. To improved the sensing performance, $\mathrm{TiO}_{2}$ nanoparticles colloid were prepared, coated with porphyrin dye and deposited as thin film. It was found that the thin film of $\mathrm{TiO}_{2}$ nanoparticles coated with porphyrin dye has more intensive interaction toward volatile organic compounds than porphyrin thin film, and improved the selective property. This may be due to the nanostructured thin film provided more surface area for dye molecules to react with VOCs.
\end{abstract}

\section{Introduction}

Volatile Organic Compounds (VOCs) are organic chemicals that easily vaporize at room temperature. VOCs are emitted as gases by a wide array of products such in paints, cleaning supplies, pesticides and building materials. Long exposure time to the VOCs cause bad health effect and cancer for human. Hence, it is very important to have a sensor for identification of the hazardous VOCs. Recently, sensor base on optical sensing technique has received growing interest as an alternative to sensors based on electrical resistance that operates at high temperature. Various techniques have been employed, which include fluorescence [1], UV-VIS absorption [2], refractive index [3] and surface plasmon resonance (SPR) [4]. Fluorescence gas sensor offers several advantages over other methods such as sensitivity, high efficiency and specificity. It has been sought for huge potential applications in food quality control [5], medical [6] and environmental [7].

Chemical dyes made of porphyrins and their related compounds were among the most prospective candidate for development of gas sensor system. These chemicals have shown good sensitivity to most volatile compounds through optical sensing techniques. Their interesting features in the interactions with the gaseous molecules have been widely described elsewhere [8]. Recently, there have been many efforts carried out to improve the response properties of the sensing element to the presence of the gaseous molecules. A most straightforward strategy is to modify the nanostructure of the sensor surface. This procedure is believed to be able to directly adjust the properties of the sensor surface, such as surface molecular density and surface roughness, which in turn improve their sensing properties [9]. One of the techniques to modify the nanostructure of the sensor surface is by incorporate nanoparticles such as $\mathrm{TiO}_{2}$ in the thin film sensing material.

This paper reports the performance of fluorescence gas sensor of porphyrin dye towards volatile organic compounds, which are ethanol, acetone and 2-propanol. The sensing sensitivity was based on the changes of photoluminescence spectra of thin film in presence of volatile organic compounds. The effect of incorporate $\mathrm{TiO}_{2}$ nanoparticles in the porphyrin thin films was studied. 


\section{Experimental}

Porphyrin dye used for sensing material was iron (III) meso-tetraphenylporphine chloride. The colloidal solution of dye in toluene was prepared at a concentration of $0.2 \mathrm{mg} / \mathrm{ml}$. Meanwhile, $\mathrm{TiO}_{2}$ nanoparticles colloid was synthesized from titanium (IV) ethoxide in ethanol with addition of kalium chloride $(\mathrm{KCl})$ as stabilizer [10] with some modifications. Firstly, ethanol was mixed with $\mathrm{KCl}$ and stirred for one hour to get a complete mixture. Then, titanium ethoxide was dropped wisely to the admixture solution in glove box under nitrogen atmosphere and humidity at $15 \%$. Acetylaceton was dropped into the solution at room temperature and stirred for two hours to form a clear, transparent yellowish solution. To prepare $\mathrm{TiO}_{2}$ nanoparticles coated with porphyrin dye, the porphyrin solution was added drop wisely into the $\mathrm{TiO}_{2}$ solution and stirred for one hour to obtain a complete mixture of porphyrin and $\mathrm{TiO}_{2}$ nanoparticles colloidal solution. The volume ratio of porphyrin to $\mathrm{TiO}_{2}$ was 2 to 1 .

The thin films of porphyrin dye and $\mathrm{TiO}_{2}$ nanoparticles coated with porphyrin were deposited onto quartz substrate using self-assembly technique. The clean quartz substrate was immersed in Poly-L-Lysine (PLL) solution for 30 minutes to charge the surface into positive charge. Then, the substrate was rinse with deionized water. Using KSV Dip coating system, the substrate was dipped into the colloidal. The substrate was kept in the colloid for 30 minutes and lifted up at a constant speed of $15 \mathrm{~mm} / \mathrm{min}$. One bilayer of porphyrin dye and $\mathrm{TiO}_{2}$ nanoparticles coated with dye thin films was formed on the substrate surface. The prepared thin films were characterized using scanning electron microscopy (SEM) to study the formation of nanoparticles and measure the size of nanoparticles. The surface morphology of thin film was recorded using atomic force microscopy (AFM). Luminescence spectrophotometer was used to measure emission spectra of the thin films.

The sensing properties of the thin films toward volatile organic compounds; ethanol, acetone and 2-propanol were studied using Perkin Elmer luminescence spectrophotometer. The experimental set-up depicted in Fig. 1. Nitrogen flow is used to carrier gas bubbling through a glass balloon flask containing $10 \mathrm{ml}$ of VOCs in airflow at rate of $16 \mathrm{ml} / \mathrm{min}$ which was control by Brook's mass flow meter controller. Then, the gases enter the analysis chamber and reach to the thin films surface, where each thin film will produce photoluminescence spectra with a distinct emission peaks.

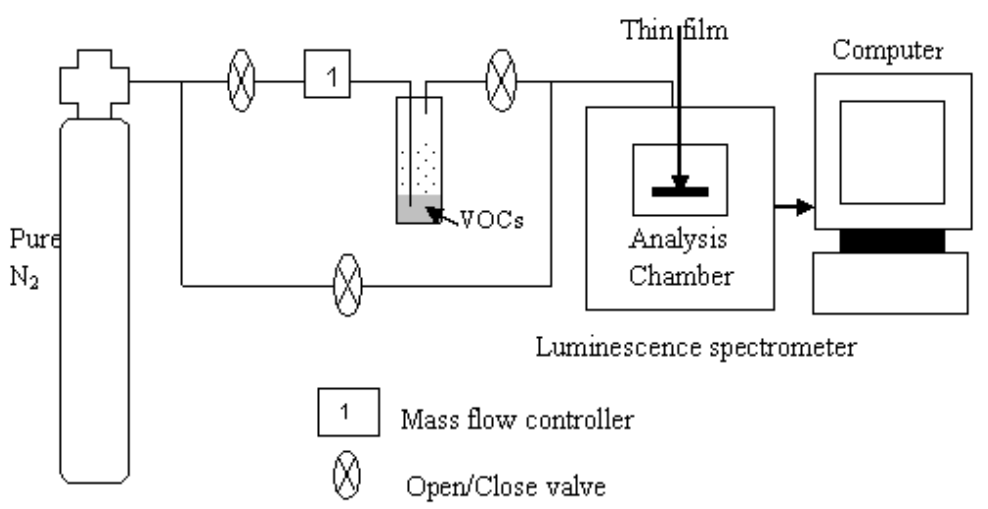

Figure1. Fluorescence gas sensing system setup.

\section{Results and Discussion}

Fig. 2 and Fig. 3 show the SEM and AFM images of the porphyrin dye and $\mathrm{TiO}_{2}$ nanoparticles coated with porphyrin thin films. The averages diameters of nanoparticles that have been synthesized and surface roughnesses obtained from these images were $20.21 \mathrm{~nm}$ and $2.167 \mathrm{~nm}$ respectively for porphyrin dye thin film, and $15.21 \mathrm{~nm}$ and $10.50 \mathrm{~nm}$ respectively for $\mathrm{TiO}_{2}$ nanoparticles coated with dye thin film. The grain size and surface roughness are important parameters to determine the sensing sensitivity of the thin film towards VOCs. 


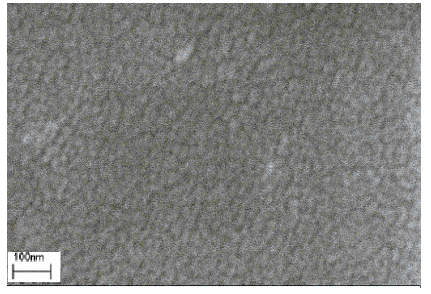

(a)

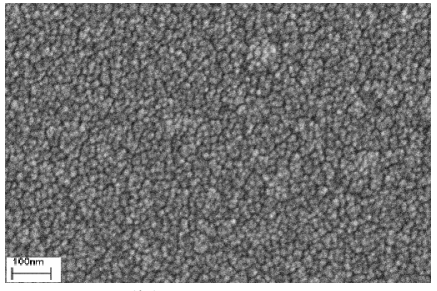

(b)

Figure 2. SEM images of (a) porphyrin dye and (b) $\mathrm{TiO}_{2}$ nanoparticles coated porphyrin dye thin films. Scale bar is $100 \mathrm{~nm}$.

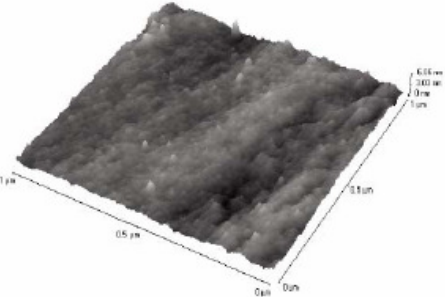

(a)

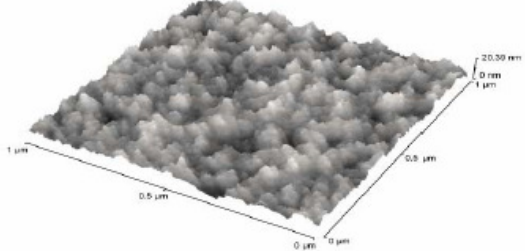

(b)

Figure 3. Three-dimensinal AFM images in the areas of $1 \mathrm{x} 1(\mu \mathrm{m})^{2}$ of (a) porphyrin dye and (b) $\mathrm{TiO}_{2}$ nanoparticles coated porphyrin dye thin films.

Fig. 4 compares the emission spectra of porphyrin dye and $\mathrm{TiO}_{2}$ nanoparticles coated porphyrin dye thin films. The emission spectrum of the thin film shifted to red with addition of $\mathrm{TiO}_{2}$ nanoparticles. When exposed to VOCs, both of the thin films undergo change in their fluorescence emission spectra (Fig. 5).

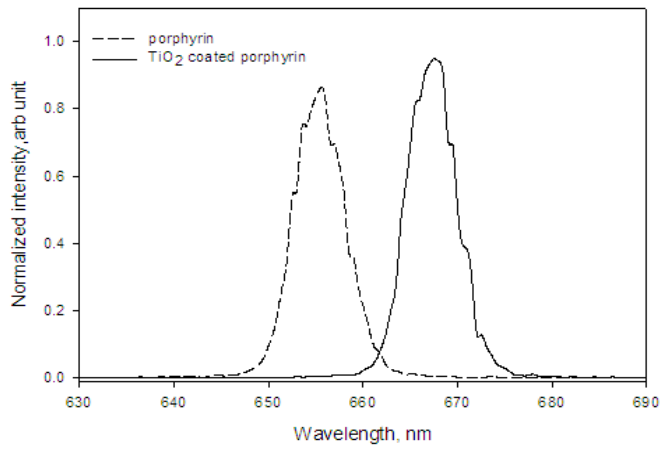

Figure 4. Emission spectra of porphyrin dye and $\mathrm{TiO}_{2}$ nanoparticles coated porphyrin dye thin films

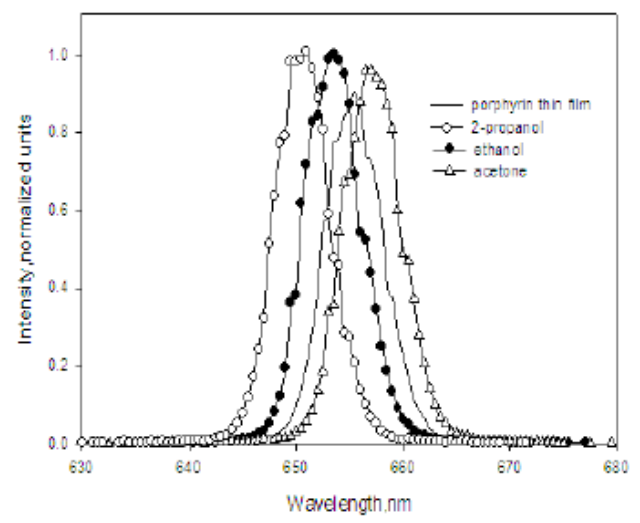

(a)

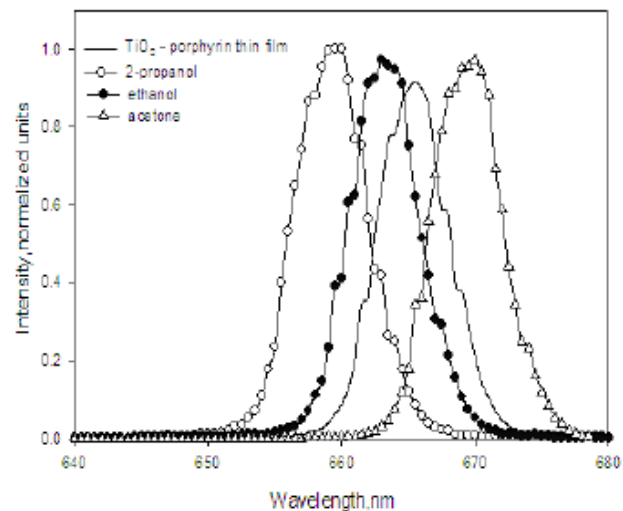

(b)

Figure 5. Emission spectra of (a) porphyrin dye and (b) $\mathrm{TiO}_{2}$ nanoparticles coated porphyrin dye thin films in presence of ethanol, 2-popanol and acetone. 
The changes of emission peak of the thin films from the original either to red shift and blue shift depended on changes in electronic states between the ground state and excited state of the porphyrin molecules. These changes could be due to the interaction of porphyrin molecules with volatile organic compounds molecules that causes rearrangement of the electrical dipole in the porphyrin compounds of the thin film [8]. A certain circumstance may be created by this activity is the changes on the energy gap between highest occupied molecular orbital (HUMO) and lowest unoccupied molecular orbital (LUMO). Thin films before interacting with VOCs molecules possesses a gap between these two orbital that determines its initial emission upon the excitation light due to the excited transition of the electrons to the ground state. When an excitation light source absorbed by the thin films molecules, which interact with gas molecules, it may emits light at different wavelength peaks.

It was found that, the thin film of $\mathrm{TiO}_{2}$ nanoparticles coated porphyrin dye shows more intensive interaction with wide selectivity property than porphyrin dye thin film. This may be due to the nanostructured thin film provided more surface area for dye molecules to react with VOCs. The sensing experiments using the same thin films were repeated for many times. It was found that no significant different of the results. This means that the sensor has repeatability property and suitable to be use as sensing material for identify type of volatile organic compounds.

\section{Summary}

Thin films of porphyrin dye and $\mathrm{TiO}_{2}$ nanoparticles coated porphyrin dye were succsessfuly fabricated using self-assembly technique. In presence of air and volatile organic compounds, the thin films produced different emission spectra and ease for chemical identification process. The thin film of $\mathrm{TiO}_{2}$ nanoparticles coated porphyrin produced more intensive interaction with wide selectivity property than the porphyrin dye thin film. Hence, it is potentially be use as fluorescence gas sensor.

\section{Acknowledgements}

The authors would like to thank the Malaysia Ministry of Science, Technology and Innovation for the grant Science Fund 03-01-02-SF0359 project.

\section{References}

[1] F. S. Mehamod, R. Daik and M. Ahmad: Sensor. and Actuat. B Vol. 96 (2003), p.537.

[2] A.A. Umar, M. M. Salleh and M. Yahaya: Curr. Appl. Phys. Vol. 8 (2008), p. 53.

[3] G. Z. Xiao, A. Adnet, Z. Zhang, F. G. Sun and C. P. Grover: Sensor. Actuat. A Vol. 118 (2005), p. 177.

[4] Rajan, S. Chand and B.D. Gupta: Sensor. Actuat. B. Vol. 123 (2007), p. 661.

[5] M.S. Kim, A.M. Lefcourt, and Y. R. Chen: Proceed. SPIE Vol. 5271 (2004), p. 62.

[6] M.Z.Krecicka, T. Krecicki, M. Fraczek, E.B. Pawlik and T.Zatonski: Otolaryngol Pol. Vol. 59 (2005), p. 195.

[7] S.W. Hong, K.H. Kim, J. Huh, C. H. Ahn, and W.H. Jo: Chem. Mater. Vol 17 (2005), p. 6213.

[8] Akrajas, M. M. Salleh and M. Yahaya: Sensor. Actuat. B Vol. 85, (2002) p. 191.

[9] A.Umar, M.M. Salleh and M. Yahaya: Eur. Phys. J. Appl. Phys. Vol. 29 (2005) p. 215.

[10] S. E. Assmann, J. Widoniak and G. Maret: Chem. Mater Vol. 16 (2004), p. 6. 